\title{
ON THE GROWTH PROPERTIES OF A FUNCTION OF TWO COMPLEX VARIABLES GIVEN BY ITS POWER SERIES EXPANSION
}

\author{
BY \\ ABE GELBART
}

1. Introduction. One of the most fundamental formulas in the theory of functions of one complex variable is the Cauchy integral formula. It is of particular value in the Weierstrass-Hadamard approach, i.e., in obtaining properties of a function from the coefficients of its power series expansion. A similar formula cannot be obtained for functions of two complex variables for an arbitrary four-dimensional domain, as is obtained, for instance, for the bicylinder, where the integration is taken over a two-dimensional surface on the boundary. Bergman $\left({ }^{1}\right)$ has shown, however, that for certain domains far more general than those previously considered, i.e., domains bounded by a finite number of analytic hypersurfaces, an analogous formula does exist, the double integral being taken essentially over the two-dimensional surface common to two or more of the analytic bounding hypersurfaces $\left({ }^{2}\right)$.

In this paper we shall obtain growth properties in terms of the coefficients of the power series expansion of a function $f\left(z_{1}, z_{2}\right)$ of two complex variables analytic in special domains of the type mentioned above; first, with the aid of Bergman's integral formula, along the two-dimensional surfaces common to the bounding hypersurfaces, and then, along a class of two-dimensional surfaces lying in only one of the bounding hypersurfaces and having a line of contact with another bounding hypersurface. We also obtain a mapping theorem which determines from the coefficients a convex region in the $f_{1} f_{2}$-plane, $f\left(z_{1}, z_{2}\right)=f_{1}+i f_{2}$, which must be contained in the smallest convex region of the mapping on the $f_{1} f_{2}$-plane of the surfaces considered.

2. Properties of $f$ associated with $G^{2}(r)$. Let us consider a finite four-dimensional domain $\mathfrak{M}^{4}$ which is bounded by the hypersurfaces

$$
\begin{aligned}
s_{1}^{3}(r) & \equiv E\left[z_{2}=r e^{i \lambda_{1}}, 0 \leqq \lambda_{1} \leqq 2 \pi\right], \\
s_{2}^{3}(r) & \equiv E\left[z_{1}=r e^{i \lambda_{2}}+p\left(\lambda_{2}\right) z_{2} \equiv h\left(\lambda_{2}, z_{2}\right), 0 \leqq \lambda_{2} \leqq 2 \pi\right],
\end{aligned}
$$

and which depends on a positive parameter $r ; p\left(\lambda_{2}\right)$ is assumed merely to have

Presented to the Society in two parts: October 28,1939, under the title On functions of two complex variables given by power series expansion, and December 27, 1939, under the title On the growth of a function of two complex variables given by its power series expansion on certain hypersurfaces; received by the editors May 28, 1940.

(1) Bergman [2, 3]. See the bibliography at the end of this paper.

(2) Bergman calls such surfaces "distinguished boundary surfaces." 
a first derivative. Let $G^{2}(r)$ be the two-dimensional surface on the boundary of $\mathfrak{M}^{4}$ which is the common part of the bounding hypersurfaces, i.e.,

$$
G^{2}(r) \equiv s_{1}^{3} \cdot s_{2}^{3}
$$

THEOREM I. Given a function $f\left(z_{1}, z_{2}\right)=\sum_{m, n=0}^{\infty} a_{m n} z_{1}^{m} z_{2}^{n}$ regular in the domain $\overline{\mathfrak{M}}^{4}(r)$; if $M(r)$ is the maximum-modulus of $f\left(z_{1}, z_{2}\right)$ on $G^{2}(r)$, then

$$
M(r) \geqq \max _{m, n} \frac{r^{m+n}\left|a_{m n}\right|}{G(m, n ; p) B(p)},
$$

where $m$ and $n$ range over all non-negative integral values, $B(p)$ is a constant depending upon $p$, and $G(m, n ; p)$ is a function of $m, n$, and $p$, given by

$$
\begin{aligned}
& 1+\int_{0}^{n+1}\left(1+x \frac{1+\log m}{m}\right)^{m} \max \left|p\left(\lambda_{2}\right)\right| x d x-\frac{m}{\log p}-\frac{m}{1+\log m}, \\
& \text { when } \max |p|<1, m \geqq 1 \text {, } \\
& 1+\int_{0}^{n+1}\left(1+x \frac{1+\log m}{m}\right)^{m} \max \left|p\left(\lambda_{2}\right)\right|^{x} d x \\
& \frac{1-\max \left|p\left(\lambda_{2}\right)\right|^{n+1}}{1-\max \left|p\left(\lambda_{2}\right)\right|}
\end{aligned}
$$

Proof of Theorem I. Keeping $z_{2}$ constant, say equal to $t_{2}$, we obtain for a particular value of $z_{1}$, say $t_{1}$,

$$
f\left(t_{1}, t_{2}\right)=\frac{1}{2 \pi i} \int_{0}^{2 \pi} \frac{f\left[h\left(\lambda_{2}, t_{2}\right), t_{2}\right]\left[i r e^{i \lambda_{2}}+p^{\prime}\left(\lambda_{2}\right) t_{2}\right] d \lambda_{2}}{\left[\left(r e^{i \lambda_{2}}+p\left(\lambda_{2}\right) t_{2}\right)-t_{1}\right]} .
$$

Since the numerator of the integrand is an analytic function of $t_{2}$, we again apply the Cauchy integral formula and obtain

$$
\begin{aligned}
f\left(t_{1}, t_{2}\right)= & \frac{1}{(2 \pi i)^{2}} \int_{0}^{2 \pi} \int_{0}^{2 \pi} \frac{f\left[h\left(\lambda_{2}, r e^{i \lambda_{1}}\right), r e^{i \lambda_{1}}\right]}{\left[\left(r e^{i \lambda_{2}}+p\left(\lambda_{2}\right) t_{2}\right)-t_{1}\right]\left[r e^{i \lambda_{1}}-t_{2}\right]} \\
& \cdot\left[i r e^{i \lambda_{2}}+p^{\prime} r e^{i \lambda_{1}}\right] i r e^{i \lambda_{1}} d \lambda_{1} d \lambda_{2} .
\end{aligned}
$$

For the $m$ th derivative of $f\left(t_{1}, t_{2}\right)$ with respect to $t_{1}$, we obtain

$$
\begin{aligned}
\frac{\partial^{m} f\left(t_{1}, t_{2}\right)}{\partial t_{1}^{m}}= & \frac{m !}{(2 \pi i)^{2}} \int_{0}^{2 \pi} \int_{0}^{2 \pi} \frac{f\left[h\left(\lambda_{2}, r e^{i \lambda_{1}}\right), r e^{i \lambda_{1}}\right]}{\left[\left(r e^{i \lambda_{2}}+p\left(\lambda_{2}\right) t_{2}\right)-t_{1}\right]^{m+1}\left[r e^{i \lambda_{1}}-t_{2}\right]} \\
& \cdot\left[i r e^{i \lambda_{2}}+p^{\prime}\left(\lambda_{2}\right) r e^{i \lambda_{1}}\right] i r e^{i \lambda_{2}} d \lambda_{1} d \lambda_{2} .
\end{aligned}
$$

Let

$$
H_{1} \equiv\left(r e^{i \lambda_{2}}+p\left(\lambda_{2}\right) t_{2}\right)-t_{1}, \quad H_{2} \equiv r e^{i \lambda_{1}}-t_{2}
$$


For the $n$th derivative of $1 / H_{1}^{m+1} H_{2}$ with respect to $t_{2}$, we obtain by Leibnitz' rule

$$
\left[1+\sum_{\nu=1}^{n} \frac{(m+\nu) !}{m ! \nu !}\left(\frac{H_{2}}{H_{1}} p\left(\lambda_{2}\right)\right)^{\nu}\right] \frac{n !}{H^{m+1} H_{2}^{n+1}} .
$$

Hence we obtain for $\partial^{m+n} f\left(t_{1}, t_{2}\right) / \partial t^{m} \partial t^{n}$ the expression

$$
\begin{aligned}
\frac{m ! n !}{(2 \pi i)^{2}} \int_{0}^{2 \pi} \int_{0}^{2 \pi} \frac{f\left[h, \lambda_{1}\right]\left[i r e^{i \lambda_{2}}+p^{\prime}\left(\lambda_{2}\right) r e^{i \lambda_{1}}\right] i r e^{i \lambda_{1}}}{\left[\left(r e^{i \lambda_{2}}+p\left(\lambda_{2}\right) t_{2}\right)-t_{1}\right]^{m+1}\left[r e^{i \lambda_{1}}-t_{2}\right]^{n+1}} \\
\cdot\left[1+\sum_{\nu=1}^{n} \frac{(m+\nu) !}{m ! \nu !}\left(\frac{H_{2}}{H_{1}} p\right)^{\nu}\right] d \lambda_{1} d \lambda_{2} .
\end{aligned}
$$

Now

Hence

$$
a_{m n}=\frac{\partial^{m+n} f(0,0)}{m ! n ! \partial t_{1}^{m} \partial t_{2}^{n}}
$$

$$
\begin{aligned}
a_{m n}= & \frac{1}{(2 \pi i)^{2}} \int_{0}^{2 \pi} \int_{0}^{2 \pi} \frac{f\left[h, \lambda_{1}\right]\left[i r e^{i \lambda_{2}}+p^{\prime} r e^{i \lambda_{1}}\right] i r e^{i \lambda_{1}}}{r^{m+n+2} \exp \left\{i\left(m \lambda_{2}+n \lambda_{1}+\lambda_{2}+\lambda_{1}\right)\right\}} \\
& \cdot\left[1+\sum_{\nu=1}^{n} \frac{(m+\nu) !}{m ! \nu !}\left(e^{i\left(\lambda_{1}-\lambda_{2}\right)} p\right)^{\nu}\right] d \lambda_{1} d \lambda_{2} .
\end{aligned}
$$

Taking the absolute value of $a_{m n}$ we get

$$
\begin{aligned}
\left|a_{m n}\right| \leqq & \frac{1}{4 \pi^{2}} \frac{M(r) \max _{0 \leqq \lambda_{2} \leqq 2 \pi}\left[1+\left|p^{\prime}\left(\lambda_{2}\right)\right|\right]}{r^{m+n}} \\
& \cdot\left[1+\sum_{\nu=1}^{n} \frac{(m+\nu) !}{m ! \nu !}\left(\max |p|^{\nu}\right)\right] 4 \pi^{2} .
\end{aligned}
$$

Now for $m \geqq 1$, it can be shown that

$$
1+\sum_{\nu=1}^{n}\left[\frac{(m+\nu) !}{m ! \nu !} \max \left|p\left(\lambda_{2}\right)\right| v\right]
$$

When $|p|<1$ we have

$$
\leqq 1+\sum_{\nu=1}^{n}\left(1+\nu \frac{1+\log m}{m}\right)^{m}|p|^{\nu} .
$$

$$
\begin{aligned}
1 & +\sum_{\nu=1}^{n} \frac{(m+\nu) !}{m ! \nu !} \max \left|p\left(\lambda_{2}\right)\right|^{\nu} \\
& \leqq 1+\int_{1}^{n}\left[1+x \frac{1+\log m}{m}\right]^{m} \max |p|^{x} d x-\frac{m}{\log p}-\frac{m}{1+\log m}
\end{aligned}
$$

and when $|p| \geqq 1$, 


$$
1+\sum_{\nu=1}^{n} \frac{(m+\nu) !}{m ! \nu !} \max |p|^{\nu} \leqq \int_{0}^{n+1}\left[1+x \frac{1+\log m}{m}\right]^{m} \max |p|^{x} d x
$$

When $m=0,\left(1-\max |p|^{n+1}\right) /(1-\max |p|)$ is the exact value of the left-hand side of (2.14) for all $p$.

Therefore for all differentiable $p\left(\lambda_{2}\right)$ and non-negative integral values of $m$ and $n$ we have

$$
\left|a_{m n}\right| \leqq \frac{M(r) B(p)}{r^{m+n}} G(m, n ; p),
$$

wherè $B(p)=\max _{0 \leqq \lambda_{2} \leqq 2 \pi}\left(1+\left|p^{\prime}\right|\right)$, or

$$
M(r) \geqq \frac{r^{m+n}\left|a_{m n}\right|}{B(p) G(m, n ; p)} .
$$

To find those values of $m$ and $n$, say $\mu(r)$ and $\nu(r)$, for which the right-hand expression in (2.16) is maximum for a given $r$, we take the logarithm of the expression, letting $-\log \left|a_{m n}\right|=g_{m n}$ and employ a generalized Newton polygon method. Then

$$
\begin{aligned}
g_{m n}-(m+n) \log r+ & \log B+\log G(m, n) \\
& \geqq g_{\mu \nu}-(\mu+\nu) \log r+\log B+\log G(\mu, \nu)=C .
\end{aligned}
$$

We choose $m, n$, and $g_{m n}$ as the $x$-, $y$-, and $z$-axes, respectively, and plot the points $\left(m, n, g_{m n}\right)$. Then the $m$ and $n$ of the first point which lies in the surface $z=x \log r+y \log r-\log G(x, y)-\log B+k$ as this surface is translated along the $z$-axis from $-\infty$ by varying $k$, i.e., until $k=C$, are the $\mu$ and $\nu$ which give the right-hand side of (2.16) a maximum. If there is more than one point lying on the surface, the one with the smaller $m$ is chosen; if the $m$ 's are the same, the one with the smaller $n$ is chosen. $\mu$ and $\nu$ are obviously functions of $r$.

We then have

$$
M(r) \geqq \frac{r^{\mu+\nu}\left|a_{\mu \nu}\right|}{B G(\mu, \nu)}
$$

This gives a lower bound for the growth of $f\left(z_{1}, z_{2}\right)$ along the hypersurface $g^{3} \equiv \mathrm{S}_{r=r_{0}}^{r_{1}} G^{2}(r)$, where $r$ varies continuously.

3. The mapping of the surface $G^{2}(r)$. Let us introduce the function

$$
F(f, \alpha)=e^{e^{-i \alpha_{f}\left(z_{1}, z_{2}\right)}}=\sum_{r, 8=0}^{\infty} A_{r g}^{r} z_{1} z_{2}
$$

where $0 \leqq \alpha \leqq 2 \pi$ and $f$ is defined as in the previous sections. The coefficients $\left\{A_{r s}\right\}$ are functions of $\alpha$ and a combination of the $a_{m n}$ 's such that $m \leqq r$ and $n \leqq s$. We define the region $R^{2}(r)$ as the product of the half-planes 


$$
f_{1} \cos \alpha+f_{2} \sin \alpha \leqq Q(\alpha, r),
$$$$
0 \leqq \alpha<2 \pi
$$

where $f_{1}$ and $f_{2}$ are cartesian coordinates in the $f_{1} f_{2}$-plane, and

$$
Q(\alpha, r) \equiv \log \left|A_{\mu \nu}(a, \alpha)\right|+(\mu+\nu) \log r-\log G(\mu, \nu)-\log B
$$

Theorem II. Let $f\left(z_{1}, z_{2}\right)=f_{1}+i f_{2}$. Then the smallest convex domain enclosing the mapping of $G^{2}(r)$ on the $f_{1} f_{2}$-plane contains the closed convex region $R^{2}(r)$ which depends only on the coefficients of the expansion of $f\left(z_{1}, z_{2}\right)$ and the surface $G^{2}(r)$.

This gives a lower bound, so to speak, of the mapping of $G^{2}(r)$ on the $f_{1} f_{2}$-plane.

Proof of Theorem II. Let

$$
P(r)=\max \left|e^{e^{-i \alpha_{f\left(z_{1}, z_{2}\right)}}}\right|
$$

on the surface $G^{2}(r)$; then from (3.4) and (2.18)

$$
\begin{aligned}
\log P(r)= & \log \left|\exp \left\{e^{-i \alpha} f^{*}\left(z_{1}, z_{2}\right)\right\}\right| \\
= & \log \left|\exp \left\{\left(f_{1}^{*} \cos \alpha+f_{2}{ }^{*} \sin \alpha\right)\right\}\right| \\
& \cdot\left|\exp \left\{-i\left(f_{1}^{*} \sin \alpha-f_{2}{ }^{*} \cos \alpha\right)\right\}\right| \\
= & f_{1}^{*} \cos \alpha+f_{2}{ }^{*} \sin \alpha \\
\geqq & \log \left|A_{\mu \nu}(a, \alpha)\right|+(\mu+\nu) \log r-\log G(\mu, \nu)-\log B=Q(\alpha, r),
\end{aligned}
$$

where the ${ }^{*}$ indicates that value of $f$ which gives $|P|$ its maximum, for a given $\alpha$. Now, for each $\alpha, Q(\alpha, r)$ has a fixed value (depending on $r$ ). It is clear from (3.6) that at least one point of the mapping, namely, $\left(f_{1}^{*}, f_{2}^{*}\right)$, will lie in the half-plane

$$
f_{1} \cos \alpha+f_{2} \sin \alpha \geqq Q(\alpha, r) .
$$

The region $R^{2}(r)$ will therefore be contained in the smallest convex domain containing the mapping of $G^{2}(r)$ on the $f_{1} f_{2}$-plane. Theorem II is then proved.

It is clear that a similar theorem will hold for any surface for which we have a lower bound for the maximum of the function $f\left(z_{1}, z_{2}\right)$ on the surface. For example, we can state similar theorems for the surfaces considered in $\S \S 4$ and 5.

4. Further properties of the function on other surfaces of the type $G^{2}(r)$. Let us consider the finite four-dimensional region $\mathfrak{M}^{4}(r)$ bounded by the three infinite hypersurfaces:

$$
\begin{aligned}
s_{1}^{3}(r) & \equiv E\left[z_{2}=r e^{i \lambda_{1}}, 0 \leqq \lambda_{1} \leqq 2 \pi\right], \\
s_{2}^{3}(r) & \equiv E\left[z_{1}=r e^{i \lambda_{2}}+C_{2} z_{2}, 0 \leqq \lambda_{2} \leqq 2 \pi\right], \\
s_{3}^{3}(r) & \equiv E\left[z_{1}=r e^{i \lambda_{3}}-C_{3} z_{2}, 0 \leqq \lambda_{3} \leqq 2 \pi\right],
\end{aligned}
$$


where, as above, $r$ is a parameter and $C_{2}$ and $C_{3}$ are positive constants less than unity. This restriction on $C_{2}$ and $C_{3}$ is necessary in order that the hypersurfaces of (3.1) form the boundary of a finite closed domain. Let $G_{k s}^{2}(r)$ be that part of $s_{k}^{3}(r) \cdot s_{s}^{3}(r)$ which belongs to the boundary of $\mathfrak{M}^{4}$. Now let

$$
G^{2}(r) \equiv G_{12}^{2}(r)+G_{13}^{2}(r)+G_{23}^{2}(r) .
$$

Let also $g^{3} \equiv \mathrm{S}_{r=r_{0}}^{r_{1}} G^{2}(r)$, and $g_{k s}^{3} \equiv \mathrm{S}_{r=r_{0}}^{r_{1}} G_{k s}^{2}(r)$, where $r$ varies continuously and $r_{1}<\infty$.

Let $f\left(z_{1}, z_{2}\right)$, as before, be an analytic function regular in $\overline{\mathfrak{M}}^{4}$. We now apply Bergman's integral formula $\left(^{3}\right)$ for functions of two complex variables which states that at a point $\left(t_{1}, t_{2}\right)$ in $\mathfrak{M}^{4}$,

$$
\begin{aligned}
f\left(t_{1}, t_{2}\right) & =\frac{1}{2} \sum_{k, s}^{\prime} M_{k s}\left(t_{1}, t_{2}\right) \\
& =\frac{1}{2(2 \pi i)^{2}} \sum_{k, s}^{\prime} \iint_{B_{k s}^{2}} \frac{f\left(\phi_{k s}^{(1)}, \phi_{k s}^{(2)}\right) B_{k s}\left(t_{1}, t_{2}, \lambda_{k}, \lambda_{s}\right) d \lambda_{k} d \lambda_{s}}{\Phi_{k}\left(t_{1}, t_{2}, \lambda_{k}\right) \Phi_{s}\left(t_{1}, t_{2}, \lambda_{s}\right)}, \\
B_{k s}\left(t_{1}, t_{2}, \lambda_{k}, \lambda_{s}\right) & =\frac{Z_{k s}\left(t_{1}, t_{2}, \lambda_{k}, \lambda_{s}\right)}{\left(\phi_{k s}^{(1)}-t_{1}\right)\left(\phi_{k s}^{(2)}-t_{2}\right)}, \quad k \neq s, \\
Z_{k s}\left(t_{1}, t_{2}, \lambda_{k}, \lambda_{s}\right) & =\frac{D\left(\phi_{k s}^{(1)}, \phi_{k s}^{(2)}\right)}{D\left(\lambda_{k}, \lambda_{s}\right)}\left[\Phi_{s}\left(t_{1}, t_{2}, \lambda_{s}\right) \Phi_{k}\left(t_{1}, \phi^{(1)}, \lambda_{k}\right)\right. \\
- & \left.-\Phi_{k}\left(t_{1}, t_{2}, \lambda_{k}\right) \Phi_{s}\left(t_{1}, \phi^{(2)}, \lambda_{s}\right)\right],
\end{aligned}
$$

where $B_{k s}^{2}$ is the surface range of integration. We have in our case

$$
\begin{aligned}
& \Phi_{1}=z_{2}-r e^{i \lambda_{1}}, \\
& \Phi_{2}=z_{1}-r e^{i \lambda_{2}}-C_{2} z_{2}, \\
& \Phi_{3}=z_{1}-r e^{i \lambda_{3}}+C_{3} z_{2} ; \\
& \left\{\begin{array}{l}
z_{1}=\phi_{12}^{(1)} \equiv r e^{i \lambda_{2}}+C_{2} r e^{i \lambda_{1}}, \\
z_{2}=\phi_{12}^{(2)} \equiv r e^{i \lambda_{1}},
\end{array}\right. \\
& \left\{\begin{array}{l}
z_{1}=\phi_{13}^{(1)} \equiv r e^{i \lambda_{2}}-C_{3} r e^{i \lambda_{1}}, \\
z_{2}=\phi_{13}^{(2)} \equiv r e^{i \lambda_{1}},
\end{array}\right. \\
& \left\{\begin{array}{l}
z_{1}=\phi_{23}^{(1)} \equiv \frac{1}{C_{2}+C_{3}}\left[C_{2} r e^{i \lambda_{3}}+C_{3} r e^{i \lambda_{2}}\right], \\
z_{2}=\phi_{23}^{(2)} \equiv \frac{1}{C_{2}+C_{3}}\left[r e^{i \lambda_{3}}-r e^{i \lambda_{2}}\right] ;
\end{array}\right.
\end{aligned}
$$

(3) Bergman [2, p. 97] and [3, p. 861]. 
and consequently,

$$
\begin{aligned}
f\left(t_{1}, t_{2}\right)= & M_{12}\left(t_{1}, t_{2}\right)+M_{13}\left(t_{1}, t_{2}\right)+M_{23}\left(t_{1}, t_{2}\right) \\
= & \frac{1}{(2 \pi i)^{2}} \iint_{B_{12}^{2}} \frac{f\left(\phi_{12}^{(1)}, \phi_{12}^{(2)}\right)\left(r^{2} e^{i\left(\lambda_{1}+\lambda_{2}\right)}\right) d \lambda_{1} d \lambda_{2}}{\left(r e^{i \lambda_{1}}-t_{2}\right)\left(r e^{i \lambda_{2}}+C_{2} t_{2}-t_{1}\right)} \\
& +\frac{1}{(2 \pi i)^{2}} \iint_{B_{13}^{2}} \frac{f\left(\phi_{13}^{(1)}, \phi_{13}^{(2)}\right)\left(r^{2} e^{i\left(\lambda_{1}+\lambda_{3}\right)}\right) d \lambda_{1} d \lambda_{3}}{\left(r e^{i \lambda_{1}}-t_{2}\right)\left(r e^{i \lambda_{3}}-C_{3} t_{2}-t_{1}\right)} \\
& +\frac{1}{(2 \pi i)^{2}} \iint_{B_{23}^{2}} \frac{f\left(\phi_{23}^{(1)}, \phi_{23}^{(2)}\right)\left(r^{2} e^{i\left(\lambda_{2}+\lambda_{2}\right)}\right) d \lambda_{2} d \lambda_{3}}{\left(r e^{i \lambda_{2}}+C_{2} t_{2}-t_{1}\right)\left(r e^{i \lambda_{3}}-C_{3} t_{2}-t_{1}\right)} .
\end{aligned}
$$

As in $\$ 2$ we have that

$$
\begin{gathered}
a_{m n}=\frac{\partial^{m+n} f(0,0)}{m ! n ! \partial t_{1}^{m} \partial t_{2}^{n}}=\left.\frac{\partial^{m+n}\left[M_{12}\left(t_{1}, t_{2}\right)+M_{13}\left(t_{1}, t_{2}\right)+M_{23}\left(t_{1}, t_{2}\right)\right]}{m ! n ! \partial t_{1}^{m} \partial t_{2}^{n}}\right|_{t_{1}, t_{2}=0}, \\
\frac{\partial^{m+n} M_{12}\left(t_{1}, t_{2}\right)}{\partial t_{1}^{m} \partial t_{2}^{n}} \\
=\frac{m !}{(2 \pi i)^{2}} \iint_{B_{12}^{2}} \frac{\partial^{n}}{\partial t_{2}^{n}}\left[\frac{f\left(\phi_{12}^{(1)}, \phi_{12}^{(2)}\right)\left(r^{2} e^{i\left(\lambda_{1}+\lambda_{2}\right)}\right)}{\left(r e^{i \lambda_{1}}-t_{2}\right)\left(r e^{i \lambda_{2}}+c_{2} t_{2}-t_{1}\right)^{m+1}}\right] d \lambda_{1} d \lambda_{2} \\
=\frac{m ! n !}{(2 \pi i)^{2}} \iint_{B_{12}^{2}} \frac{f\left(\phi_{12}^{(1)}, \phi_{12}^{(2)}\right)\left(r^{2} e^{i\left(\lambda_{1}+\lambda_{2}\right)}\right) d \lambda_{1} d \lambda_{2}}{\left[-\Phi_{1}\left(t_{2}\right)\right]^{n+1}\left[-\Phi_{2}\left(t_{1}, t_{2}\right)\right]^{m+1}},
\end{gathered}
$$

so that

$$
\begin{aligned}
\frac{\partial^{m+n} M_{12}(0,0)}{\partial t_{1}^{m} \partial t_{2}^{n}}= & \frac{m ! n !}{(2 \pi i)^{2}} \iint_{B_{12}^{2}} \frac{f\left(\phi_{12}^{(1)}, \phi_{12}^{(2)}\right)\left(r^{2} e^{i\left(\lambda_{1}+\lambda_{2}\right)}\right)}{r^{m+n+2} e^{i\left(m \lambda_{2}+n \lambda_{1}+\lambda_{2}+\lambda_{1}\right)}} \\
& \cdot \sum_{\nu=0}^{n} \frac{(m+\nu) !}{m ! \nu !}\left(c_{2} e^{i\left(\lambda_{1}-\lambda_{2}\right)}\right) \nu d \lambda_{1} d \lambda_{2} .
\end{aligned}
$$

This yields, by a process analogous to that used in $\S 2$,

$$
\frac{1}{m ! n !}\left|\frac{\partial^{m+n} M_{12}(0,0)}{\partial t_{1}^{m} \partial t_{2}^{n}}\right| \leqq \frac{B_{12}\left(g_{12}^{3}\right) M(r) G_{12}(m, n)}{r^{m+n}},
$$

where $M(r)$ is the maximum-modulus of $f$ on $G^{2}(r), B\left(g_{12}^{3}\right)$ is a constant depending on the hypersurface $g_{12}^{3}=\mathrm{S}_{r=r_{0}}^{r_{1}} G_{12}^{2}(r)$ and $G_{12}(m, n)$ is a function of $m$ and $n$, also depending on $g_{12}^{3}$ and is defined in a way similar to $G(m, n)$ of $\S 2$.

In the same way we obtain

$$
\frac{1}{m ! n !}\left|\frac{\partial^{m+n} M_{13}(0,0)}{\partial t_{1}^{m} \partial t_{2}^{n}}\right| \leqq \frac{B_{13}\left(G_{13}^{2}\right) M(r) G_{13}(m, n)}{r^{m+n}} .
$$


From (4.3), we have

$$
\Phi_{2}\left(t_{1}, t_{2}\right)=\left(t_{1}-r e^{i \lambda_{2}}-C_{2} t_{2}\right), \quad \Phi_{3}\left(t_{1}, t_{2}\right)=\left(t_{1}-r e^{i \lambda_{3}}+C_{3} t_{2}\right) .
$$

Hence

$$
\frac{\partial^{m}}{\partial t_{1}^{m}}\left[\frac{1}{\Phi_{2} \Phi_{3}}\right]=(-1)^{m} m ! \sum_{\nu=0}^{m} \frac{1}{\Phi_{2}^{\nu+1} \Phi_{3}^{m-\nu+1}},
$$

and

$$
\begin{aligned}
\frac{\partial^{m}}{\partial t_{1}^{m}}\left[\frac{1}{\Phi_{2} \Phi_{3}}\right]= & (-1)^{m+n} m ! n ! \sum_{\nu=0}^{m} \sum_{\mu=0}^{n} \frac{(m+n-\nu-\mu) ! C_{2}^{\mu} C_{3}^{n-\mu}}{(m-\nu) !(n-\mu) ! \Phi_{2}^{\nu+\mu+1} \Phi_{3}^{m+n-\nu-\mu+1}} \\
= & \frac{(-1)^{m+n} m ! n !}{\Phi_{3}^{m+n+2}} \sum_{\mu=0}^{n} \frac{1}{(n-\mu) !}\left(\frac{C_{2}}{C_{3}} \frac{\Phi_{3}}{\Phi_{2}}\right)^{\mu} \\
& \cdot \sum_{\nu=0}^{m} \frac{(m+n-\nu-\mu) !}{(m-\nu) !}\left(\frac{\Phi_{3}}{\Phi_{2}}\right)^{\nu} .
\end{aligned}
$$

Then

$$
\begin{aligned}
\frac{1}{m ! n !}\left|\frac{\partial^{m+n} M_{23}(0,0)}{\partial t_{1}^{m} \partial t_{2}^{n}}\right| \leqq & \frac{1}{4 \pi^{2}} \iint_{B_{23}^{2}} \frac{\left|f\left(\phi_{23}^{(1)}, \phi_{23}^{(2)}\right)\right|}{r^{m+n}} \sum_{\mu=0}^{n} \frac{1}{(n-\mu) !}\left(\frac{C_{2}}{C_{3}}\right)^{\mu} \\
& \cdot \sum_{\nu=0}^{m} \frac{(m+n-\nu-\mu) !}{(m-\nu) !} d \lambda_{2} d \lambda_{3} \\
\leqq & \frac{B_{23}\left(G_{23}^{2}\right) M(r)}{r^{m+n}} \sum_{\mu=0}^{n} \frac{1}{(n-\mu) !}\left(\frac{C_{2}}{C_{3}}\right)^{\mu} \\
& \cdot \sum_{\nu=0}^{m} \frac{(m+u-\nu-\mu) !}{(m-\nu) !}
\end{aligned}
$$

The constant $B_{23}\left(g_{23}^{3}\right)$ is given by $\left(1 / 4 \pi^{2}\right) \iint_{B_{23}^{2}} d \lambda_{2} d \lambda_{3}$, where the precise limits of integration are obtained by a tedious process and can be omitted here since they are not necessary for our purpose; it may be noted, however, that $0<B_{23}<1$. We shall denote by $G_{23}(m, n)$ the expression

$$
\sum_{\mu=0}^{n} \frac{1}{(n-\mu) !}\left(\frac{C_{2}}{C_{3}}\right)^{\mu} \sum_{\nu=0}^{m} \frac{(m+n-\nu-\mu) !}{(m-\nu) !} .
$$

This gives

$$
\begin{aligned}
\left|a_{m n}\right| & \leqq \frac{1}{m ! n !}\left(\left|\frac{\partial^{m+n} M_{12}(0,0)}{\partial t_{1}^{m} \partial t_{2}^{n}}\right|+\left|\frac{\partial^{m+n} M_{13}(0,0)}{\partial t_{1}^{m} \partial t_{2}^{n}}\right|+\left|\frac{\partial^{m+n} M_{23}(0,0)}{\partial t_{1}^{m} \partial t_{2}^{n}}\right|\right) \\
& \leqq \frac{M(r)}{r^{m+n}} \frac{1}{2} \sum_{k, 8=1}^{3} B_{k s}\left(g_{k s}^{3}\right) G_{k s}(m, n) .
\end{aligned}
$$


Therefore

$$
M(r) \geqq \frac{r^{m+n}\left|a_{m n}\right|}{\frac{1}{2} \sum_{k, 8=1}^{\prime 3} B\left(g_{k s}\right) G_{k s}(m, n)} .
$$

Those values of $\mu$ and $\nu$ which make the right-hand side of (4.19) a maximum can be obtained by a process similar to that employed in $\$ 2$. Hence

$$
M(r) \geqq \frac{r^{\nu+\mu}\left|a_{\mu \nu}\right|}{\frac{1}{2} \sum_{k, 8=1}^{\prime 3} B_{k s}\left(g_{k s}^{3}\right) G_{k s}(\mu, \nu)} .
$$

We can then state

THEOREM III. Given a function

$$
f\left(z_{1}, z_{2}\right)=\sum_{m, n=0}^{\infty} a_{m n} z_{1}^{m} z_{2}^{n}
$$

regular in the closed domain $\overline{\mathfrak{M}}^{4}(r)$; then along

$$
\begin{aligned}
g^{3} & =\underset{r=r_{0}}{r_{1}} G^{2}(r)=\underset{r=r_{0}}{r_{1}}\left(G_{12}^{2}(r)+G_{13}^{2}(r)+G_{23}^{2}(r)\right), \\
M(r) & \geqq \frac{r^{\nu+\mu}\left|a_{\mu \nu}\right|}{\frac{1}{2} \sum_{, 8=1}^{\prime 3} B_{k s}\left(g_{k s}^{3}\right) G_{k s}(\mu, \nu)} .
\end{aligned}
$$

5. Properties for the function on certain classes of surfaces lying in the boundary and different from $G^{2}(r)$. We next wish to consider the growth of the function $f\left(z_{1}, z_{2}\right)$ over a special class of surfaces $H^{2}(r)$ belonging to the boundary of $\mathfrak{M}^{4}$. Let

$$
H^{2}(r) \equiv E\left[z_{1}=\zeta\left(r, \lambda_{1}, \sigma\right), z_{2}=r e^{i \lambda_{1}}, \lambda_{1}^{(1)} \leqq \lambda_{1} \leqq \lambda_{1}^{(2)}, \sigma_{1} \leqq \sigma \leqq \sigma_{2}\right]
$$

where for all $\sigma$ satisfying $\sigma_{1} \leqq \sigma<\sigma_{2}$, and for any fixed $\lambda_{1}$ in the range considered,

$$
\begin{aligned}
\zeta\left(r, \lambda_{1}, \sigma\right) & \in \Im_{1}^{2}\left(r, \lambda_{1}\right), \\
J_{1}^{2}\left(r, \lambda_{1}\right) & \equiv A_{1}^{2}\left(r, \lambda_{1}\right) \cdot A_{2}^{2}\left(r, \lambda_{1}\right) \\
A_{1}^{2}\left(r, \lambda_{1}\right) & \equiv E\left[\left|z_{1}-C_{2} z_{2}\right| \leqq r, z_{2}=r e^{i \lambda_{1}}\right] \\
A_{2}^{2}\left(r, \lambda_{1}\right) & \equiv E\left[\left|z_{1}+C_{3} z_{2}\right| \leqq r, z_{2}=r e^{i \lambda_{1}}\right]
\end{aligned}
$$

and for $\sigma=\sigma_{2}$, with $\lambda_{1}$ again fixed,

$$
\zeta\left(r, \lambda_{1}, \sigma_{2}\right) \in s_{1}^{1}\left(r, \lambda_{1}\right),
$$

where $s_{1}$ is the boundary of $J_{1}^{2}\left(r, \lambda_{1}\right)$. It will be assumed that the set of all points of $H^{2}(r)$ for which $\lambda_{1}$ has an arbitrary fixed value in the range consid. 
ered is a continuous curve $h^{1}(r)\left(^{4}\right)$ with an initial point $z_{1}=\zeta\left(r, \lambda_{1}, \bar{\sigma}_{1}\left(\lambda_{1}\right)\right)$, and a terminal point on $s_{1}^{1}\left(r, \lambda_{1}\right)$. The surface $H^{2}(r)$ lies completely in that part of $s_{1}^{3}(r)$ which belongs to the boundary of the $\mathfrak{M}^{4}(r)$ of the previous section. A portion of the boundary of $H^{2}(r)$ lies on $G^{2}(r)$ of (4.2).

Let the maximum-modulus of $f\left(z_{1}, z_{2}\right)$ on $H^{2}(r)$ be $\gamma(r)$. We now map (using for simplicity the same notation for the mapped region) $J_{1}^{2}$ in to the unit circle so that $z_{1}=0$ goes into itself and the direction of the real axis, $R\left(z_{1}\right)=0$, at the point $z_{1}=0$, remains unchanged. The curve $h^{1}(r)$ maps into a segment of a continuous curve, its initial point determined by $\sigma=\bar{\sigma}_{1}\left(\lambda_{1}\right)$ and its terminal point lies on the unit circle. Now let $\theta=\left|z_{1}\right|=\left|\zeta\left(r, \lambda_{1}, \bar{\sigma}_{1}\left(\lambda_{1}\right)\right)\right|$ for $\lambda_{1}^{(1)} \leqq \lambda_{1} \leqq \lambda_{1}^{(2)}$. The quantities $\theta$ and $\alpha=\lambda_{1}^{(2)}-\lambda_{1}^{(1)}$ were introduced by Bergman and are the characteristic numbers of the surface $\left({ }^{5}\right)$.

One form of the Milloux theorem is $\left({ }^{6}\right)$ : Let $J$ be a continuous finite arc lying in the unit circle $|z| \leqq 1$ joining a point $z_{0}$ within the circle to a point on the boundary. Let $W(z)$ be regular, single-valued, and $|W(z)|<1$ inside the unit circle, and let $|W(z)| \leqq \omega$ on $J$. Then

$$
|W(0)|<\omega^{(2 / \pi) \sin ^{-1}\left(1-\theta^{\prime}\right) /\left(1+\theta^{\prime}\right)},
$$

where $\theta^{\prime}=\left|z_{0}\right|$.

Using this theorem for the mapped region $J_{1}^{2}$ with

$$
W\left(z_{1}\right)=\frac{f\left(z_{1}, z_{2}^{*}\right)}{M(r)},
$$

we have

$$
\left|W\left(z_{1}\right)\right|=\frac{\left|f\left(z_{1}, z_{2}^{*}\right)\right|}{M(r)} \leqq \frac{\gamma(r)}{M(r)}<1,
$$

and get, letting $\Theta=(2 / \pi) \sin ^{-1}(1-\theta) /(1+\theta)$,

$$
\left|f\left(0, z_{2}\right)\right|<M^{1-\Theta} \gamma^{\Theta}
$$

where $M(r)$ is the maximum-modulus of $f\left(z_{1}, z_{2}\right)$ in $\overline{\mathfrak{M}}^{4}, \lambda_{1}^{*}$ is an arbitrarily chosen value of $\lambda_{1}$ in the range considered, and $z_{2}=z_{2}^{*}=r e^{i \lambda_{1}^{*}}$.

Now

$$
\begin{aligned}
a_{0 n}=\frac{1}{2 \pi i}\left\{\int_{0}^{\lambda_{1}^{(1)}} \frac{f\left(0, r e^{i \lambda_{1}}\right)}{r^{n} e^{i n \lambda_{1}}} d \lambda_{1}+\int_{\lambda_{1}^{(1)}}^{\lambda_{1}^{(2)}} \frac{f\left(0, r e^{i \lambda_{1}}\right)}{r^{n} e^{i n \lambda_{1}}} d \lambda_{1}\right. \\
\left.\quad+\int_{\lambda_{1}^{(2)}}^{2 \pi} \frac{f\left(0, r e^{i \lambda_{1}}\right)}{r^{n} e^{i n \lambda_{1}}} d \lambda_{1}\right\},
\end{aligned}
$$

(4) The restriction that $h^{1}(r)$ be continuous is not essential since theorems of the Milloux type hold for more general one-dimensional sets.

(5) Bergman [1, pp. 347-348, Corollary]; and [4, pp. 200-201].

(6) R. Nevanlinna [5]. 


$$
\begin{aligned}
\left|a_{0 n}\right| \leqq \frac{1}{2 \pi}\left\{\int_{0}^{\lambda_{1}^{(1)}} \frac{\left|f\left(0, r e^{i \lambda_{1}}\right)\right|}{r^{n}} d \lambda_{1}+\int_{\lambda_{1}^{(1)}}^{\lambda_{1}^{(2)}} \frac{\left|f\left(0, r e^{i \lambda_{1}}\right)\right|}{r^{n}} d \lambda_{1}\right. \\
\left.+\int_{\lambda_{1}^{(2)}}^{2 \pi} \frac{\left|f\left(0, r e^{i \lambda_{1}}\right)\right|}{r^{n}} d \lambda_{1}\right\},
\end{aligned}
$$

and

$$
\left|a_{0 n}\right| \leqq \frac{1}{2 \pi r^{n}}\left[(2 \pi-\alpha) \bar{M}+\alpha M\left(\frac{\gamma}{M}\right)^{\Theta}\right],
$$

where $\bar{M}=\max \left|f\left(0, z_{2}\right)\right|=\max \left|\sum_{\nu=0}^{\infty} a_{0 \nu} z_{2}^{\nu}\right|$. Then

$$
\frac{2 \pi}{\alpha}\left[\frac{\left|a_{0 n}\right| r^{n}}{M}-\frac{\bar{M}}{M}\right]+\frac{\bar{M}}{M} \leqq\left(\frac{\gamma}{M}\right)^{\Theta} .
$$

Let $\Delta$ be defined by the equation

$$
\Delta=1-\frac{\left|a_{0 \mu}\right| r^{\mu}}{\bar{M}}
$$

$\mu$ being that $n$ which maximizes $\left|a_{0 n}\right| r^{n}$, and $\mu$ depends on $r$. Then $\Delta$ is positive.

If $\alpha>2 \pi \Delta$, we have that

$$
\gamma(r) \geqq M(r)\left[\frac{\bar{M}}{M}\left(1-\frac{2 \pi}{\alpha} \Delta\right)\right]^{\Theta^{-1}(\theta)},
$$

where the right-hand side is positive.

From these results we can state

ThEOREM IV. Given the function

$$
f\left(z_{1}, z_{2}\right)=\sum_{m, n=0}^{\infty} a_{m n} z_{1}^{m} z_{2}^{n}
$$

regular in $\overline{\mathfrak{M}}^{4}(r)$. Let $\max \left|f\left(z_{1}, z_{2}\right)\right| \leqq \gamma(r)$ on the surface $H^{2}(r)$ of (5.1) having the characteristic numbers $\theta(r)$ and $\alpha, \alpha=\lambda_{1}^{(2)}-\lambda_{1}^{(1)}>2 \pi \Delta$, where $\Delta \equiv 1-\left|a_{0} \mu\right| r^{\mu} / \bar{M}$; then

$$
\gamma(r) \geqq M(r)\left[\frac{\bar{M}(r)}{M(r)}\left(1-\frac{2 \pi \Delta}{\alpha}\right)\right]^{\Theta^{-1}(\theta)},
$$

where $M=\max \left|f\left(z_{1}, z_{2}\right)\right|$ and $\bar{M}=\max \left|f\left(0, z_{2}\right)\right|$.

Since

$$
\frac{\left|a_{0 \mu}\right| \rho^{\mu+1}}{\rho-r}>\bar{M}(r), \quad \rho>r
$$


a lower bound for $\gamma(r)$ can be obtained in terms of the coefficients of $f\left(z_{1}, z_{2}\right)$ by replacing in (5.12) $M(r)$ by the right-hand side of (4.20), $\bar{M}(r)$ by $\left|a_{0 \mu}\right| r^{\mu}$, and the $\bar{M}(r)$ in $\Delta$ by

$$
\frac{\left|a_{0 \mu^{\prime}}\right| \rho_{1}^{\mu^{\prime}+1}}{\rho_{1}-r}
$$

where

$$
\left|a_{0 \mu^{\prime}}\right| \rho_{1}^{\mu^{\prime}}=\underset{t+\epsilon<\rho<\infty}{\log . \mathrm{b}^{\prime}}\left[\max _{n}\left|a_{0 n}\right| \rho^{n}\right]
$$

for an arbitrary positive $\epsilon . \rho_{1}$ is a function of $r$ and of the coefficients $\left\{a_{0 n}\right\}$, and can be determined by a process similar to the Newton polygon method.

\section{REFERENCES}

1. St. Bergman, Zwei Sätze aus dem Ideenkreis des Schwarzschen Lemmas über die Funktionen von zwei komplexen Veränderlichen, Mathematische Annalen, vol. 109 (1934), pp. 324348 .

2. der Funktionen zweier komplexer Variabler, Mathematische Zeitschrift, vol. 39 (1934), pp. 76-79, 605-608.

3. - Über eine Integraldarstellung von Funktionen zweier komplexer Veränderlichen, Recueil Mathématique (Mathematičeski Sbornik), vol. 1 (43) (1936), pp. 851-862.

4. —_ Über eine Abschätzung von meromorphen Funktionen zweier komplexer Veränderlicher in Bereichen mit ausgezeichneter Randfläche, Travaux de l'Institut Mathématique de Tbilissi, vol. 1 (1937), pp. 187-204.

5. R. Nevanlinna, Eindeutige analytische Funktionen, chap. $4, \S 5$.

Massachusetts Institute of Technology, Cambridge, Mass. 